\section{Absence of Islet Autoantibodies and Modestly Raised Glucose Values at Diabetes Diagnosis Should Lead to Testing for MODY: Lessons From a 5-Year Pediatric Swedish National Cohort Study}

Diabetes Care 2020;43:82-89 | https://doi.org/10.2337/dc19-0747
Annelie Carlsson, ${ }^{1}$ Maggie Shepherd, ${ }^{2}$ Sian Ellard, ${ }^{2,3}$ Michael Weedon, ${ }^{2}$ Åke Lernmark, ${ }^{1}$ Gun Forsander, ${ }^{4,5}$ Kevin Colclough, ${ }^{3}$ Qefsere Brahimi, ${ }^{1}$ Camilla Valtonen-Andre, ${ }^{6}$ Sten A. Ivarsson, ${ }^{1}$ Helena Elding Larsson, ${ }^{1}$ Ulf Samuelsson, ${ }^{7,8}$ Eva Örtqvist, ${ }^{9}$ Leif Groop, ${ }^{10}$ Johnny Ludvigsson, ${ }^{7,8}$ Claude Marcus, ${ }^{11}$ and Andrew T. Hattersley ${ }^{2}$

\section{OBJECTIVE}

Identifying maturity-onset diabetes of the young (MODY) in pediatric populations close to diabetes diagnosis is difficult. Misdiagnosis and unnecessary insulin treatment are common. We aimed to identify the discriminatory clinical features at diabetes diagnosis of patients with glucokinase (GCK), hepatocyte nuclear factor1A (HNF1A), and HNF4A MODY in the pediatric population.

\section{RESEARCH DESIGN AND METHODS}

Swedish patients ( $n=3,933$ ) aged 1-18 years, diagnosed with diabetes May 2005 to December 2010, were recruited from the national consecutive prospective cohort Better Diabetes Diagnosis. Clinical data, islet autoantibodies (GAD insulinoma antigen-2, zinc transporter 8, and insulin autoantibodies), HLA type, and C-peptide were collected at diagnosis. MODY was identified by sequencing GCK, HNF1A, and HNF4A, through either routine clinical or research testing.

\section{RESULTS}

The minimal prevalence of MODY was $1.2 \%$. Discriminatory factors for MODY at diagnosis included four islet autoantibody negativity $(100 \%$ vs. $11 \%$ not-known MODY; $\left.P=2 \times 10^{-44}\right), \mathrm{HbA}_{1 \mathrm{c}}\left(7.0 \%\right.$ vs. $\left.10.7 \%[53 \mathrm{vs.} 93 \mathrm{mmol} / \mathrm{mol}] ; P=1 \times 10^{-20}\right)$, plasma glucose (11.7 vs. $26.7 \mathrm{mmol} / \mathrm{L} ; P=3 \times 10^{-19}$ ), parental diabetes ( $63 \%$ vs. $12 \%$; $\left.P=1 \times 10^{-15}\right)$, and diabetic ketoacidosis (0\% vs. $\left.15 \% ; P=0.001\right)$. Testing 303 autoantibody-negative patients identified 46 patients with MODY (detection rate 15\%). Limiting testing to the 73 islet autoantibody-negative patients with $\mathrm{HbA}_{1 \mathrm{c}}<7.5 \%(58 \mathrm{mmol} / \mathrm{mol})$ at diagnosis identified 36 out of $46(78 \%)$ patients with MODY (detection rate $49 \%$ ). On follow-up, the 46 patients with MODY had excellent glycemic control, with an $\mathrm{HbA}_{1 \mathrm{c}}$ of $6.4 \%(47 \mathrm{mmol} / \mathrm{mol})$, with 42 out of $46(91 \%)$ patients not on insulin treatment.

\section{CONCLUSIONS}

At diagnosis of pediatric diabetes, absence of all islet autoantibodies and modest hyperglycemia $\left(\mathrm{HbA}_{1 \mathrm{c}}<7.5 \%\right.$ [58 mmol/mol]) should result in testing for $\mathrm{GCK}$, HNF1A, and HNF4A MODY. Testing all $12 \%$ patients negative for four islet autoantibodies is an effective strategy for not missing MODY but will result in a lower detection rate. Identifying MODY results in excellent long-term glycemic control without insulin.

\footnotetext{
${ }^{1}$ Department of Clinical Sciences, Lund University/ Clinical Research Centre, Skåne University Hospital, Malmö, Sweden

${ }^{2}$ Institute of Biomedical and Clinical Science, University of Exeter Medical School, Exeter, U.K. ${ }^{3}$ Molecular Genetics, Royal Devon and Exeter NHS Foundation Trust, Exeter, U.K.

${ }^{4}$ The Queen Silvia Children's Hospital, Sahlgrenska University Hospital, Gothenburg, Sweden

${ }^{5}$ Institute of Clinical Sciences, University of Gothenburg, Gothenburg, Sweden

${ }^{6}$ Department of Clinical Chemistry, University and Regional Laboratories Region Skåne, Malmö, Sweden

${ }^{7}$ Crown Princess Victoria's Children's and Youth Hospital, University Hospital, Linköping, Sweden ${ }^{8}$ Department of Clinical and Experimental Medicine, Linköping University, Linköping, Sweden ${ }^{9}$ Pediatric Endocrinology Unit, Department of Women's and Children's Health, Karolinska Institute, Stockholm, Sweden

${ }^{10}$ Finnish Institute for Molecular Medicine, Helsinki University, Helsinki, Finland

${ }^{11}$ Division of Pediatrics, Department of Clinical Science Intervention and Technology, Karolinska Institute, Stockholm, Sweden
}

Corresponding authors: Claude Marcus, claude .marcus@ki.se, and Andrew T. Hattersley, a.t .hattersley@exeter.ac.uk

Received 15 April 2019 and accepted 19 October 2019

This article contains Supplementary Data online at http://care.diabetesjournals.org/lookup/suppl/ doi:10.2337/dc19-0747/-/DC.

This article is featured in a podcast available at http://www.diabetesjournals.org/content/ diabetes-core-update-podcasts.

A.C. and M.S. contributed equally to this work. J.L., C.M., and A.T.H. contributed equally to this work.

(C) 2019 by the American Diabetes Association. Readers may use this article as long as the work is properly cited, the use is educational and not for profit, and the work is not altered. More information is available at http://www. diabetesjournals .org/content/license. 
Maturity-onset diabetes of the young (MODY) is a monogenic, dominantly inherited diabetes that is typically diagnosed young but is not insulin dependent. Recognizing MODY is important, as treatment and management are different from those for type 1 and type 2 diabetes. Diabetes in children is predominantly type 1 diabetes, but type 2 diabetes and MODY also occur $(1,2)$. MODY accounts for $1-4 \%$ of pediatric diabetes (1,3-7), but misdiagnosis results in many young people being treated unnecessarily with insulin $(1,4)$, with many years' delay from initial diabetes diagnosis to correct genetic diagnosis (8).

The most common subtypes of MODY are glucokinase (GCK) MODY, which needs no treatment, and hepatocyte nuclear factor-1 $\alpha$ (HNF1A) MODY and HNF4A MODY, which are both optimally treated with low-dose sulphonylureas when pharmaceutical therapy is needed $(2,9)$.

Identifying MODY in pediatric populations with diabetes is diagnostically difficult, as no single criterion or combination of commonly used clinical criteria can adequately separate them from type 1 and type 2 diabetes $(1,2,10,11)$. This is particularly true close to diagnosis, when those with type 1 diabetes continue to produce endogenous insulin. Increasing obesity in all children (12) can also make differential diagnosis from type 2 diabetes challenging (13).

Islet autoantibodies can be useful in identifying non-type 1 diabetes and are rarely detected in MODY, being present in only $1 \%$ of cases, similar to the healthy population (14). In contrast, islet autoantibodies are detected in $\sim 90 \%$ of children with type 1 diabetes at diagnosis $(15,16)$. Despite this, use of islet autoantibodies is not universally advocated, and comprehensive islet autoantibody testing of all four subtypes-antibodies against GAD (GADA), insulinoma antigen-2 (IA$2 A)$, zinc transporter 8 (ZnT8A), and insulin (IAA) - is not routinely performed in clinical care.

A correct diagnosis of MODY in children and adolescents leads to improved treatment with the avoidance of insulin, no deterioration in $\mathrm{HbA}_{1 \mathrm{c}}(17,18)$, and cost savings (19). Making the genetic diagnosis as close as possible to the diabetes diagnosis will reduce delays in starting recommended treatment. Approaches to the recognition of MODY are currently predominantly based on clinical features at follow-up rather than at diagnosis (20).
The aim of our study was to identify the discriminatory clinical features of the most common types of MODY at diagnosis of diabetes in a pediatric national cohort.

\section{RESEARCH DESIGN AND METHODS}

Individuals aged between 1 and 18 years with a new diagnosis of diabetes were recruited from the national consecutive prospective cohort Better Diabetes Diagnosis (BDD) study, involving 42 hospital pediatric clinics in Sweden, from May 2005 to December 2010 (21). A total of 4,574 children and young people between 1 and 18 years of age were diagnosed with diabetes during the study period, and 3,933 (86\%) were recruited into the study cohort (Fig. 1). Participants were $45 \%$ female $(n=1,755)$ and had a mean age of diagnosis of 10.1 years. Patients with incidental hyperglycemia or impaired fasting glucose and not thought to have diabetes were not included in the BDD study.

\section{Clinical Characteristics}

Clinical data including symptoms of polyuria, polydipsia, and weight loss, family history of diabetes, and samples for plasma glucose concentration, $\mathrm{HbA}_{1 \mathrm{c}}$, islet autoantibodies GADA, IA-2A, ZnT8A, and IAA, HLA type, and C-peptide were collected at diagnosis. The routine laboratory tests, plasma glucose, $\mathrm{pH}$, and $\mathrm{HbA}_{1 \mathrm{c}}$ were analyzed locally, with results returned within $24 \mathrm{~h}$, and are described as early investigations (Supplementary Table 1). Diabetic ketoacidosis (DKA) was defined as $\mathrm{pH}<7.3$ or serum bicarbonate $<15 \mathrm{mEq} / \mathrm{L}$ with a plasma glucose $>11 \mathrm{mmol} / \mathrm{L}$. Blood samples sent to the reference laboratory for analyses of all islet autoantibodies (GADA, IA-2A, ZnT8A, and IAA), HLA genotype, and random C-peptide are described as delayed, as the results were returned to the clinician within 14 to 90 days (Supplementary Table 1). Demographic data, symptoms, physical signs, and blood analysis at onset were registered in SWEDIABKIDS, a national incidence and longitudinal quality control register for children and adolescents with diabetes (22).

Molecular genetic testing was undertaken to identify the most common causes of MODY (GCK, HNF1A, and HNF4A), all of which need different treatment from type 1 or type 2 diabetes. The molecular genetic sequencing of the whole coding region and critical noncoding regions of each gene was either performed because of a clinical request or as a research test at the diagnostic laboratory at the Royal Devon and Exeter NHS Foundation Trust (www.diabetesgenes.org) or at the Department of Clinical Chemistry, Division of Laboratory Medicine, Skåne University Hospital. Clinical referrals for genetic testing were predominantly requested in autoantibody-negative patients (76 out of 81 [94\%]) (Fig. 1).

Of those who were islet autoantibody negative, there were a total of 386 out of 462 patients who were not tested clinically. To assess if case subjects with MODY had been missed, which would alter treatment, research sequencing of the HNF1A, HNF4A, and GCK genes was undertaken in an additional 404 patients (227 autoantibody negative and 177 autoantibody positive). We research tested all 227 autoantibody-negative patients in whom there was sufficient DNA for genetic sequencing. There were 159 islet autoantibody-negative patients who were not tested on a clinical or research basis, as there was insufficient DNA available (Fig. 1). We compared the characteristics of the 159 islet autoantibody-negative patients not research tested with the 227 researchtested patients (Supplementary Table $2)$. No variables were significant after correction for multiple testing. Therefore, the patients research tested were representative of the whole group who were islet autoantibody negative and not tested clinically.

To provide a random collection of autoantibody-positive patients, we identified 250 consecutive patients. Research sequencing was performed on the 177 of these islet autoantibody-positive patients who had not been tested clinically for whom we had an adequate quantity of DNA for genetic testing. We compared the characteristics of the randomly selected 177 autoantibody-positive patients who were research tested for MODY to the 3,294 antibody-positive patients who were not tested (Supplementary Table 3). No variables except C-peptide were significant after correction for multiple testing. Therefore, the patients research tested were representative of the whole group who were islet autoantibody positive and not tested clinically. 


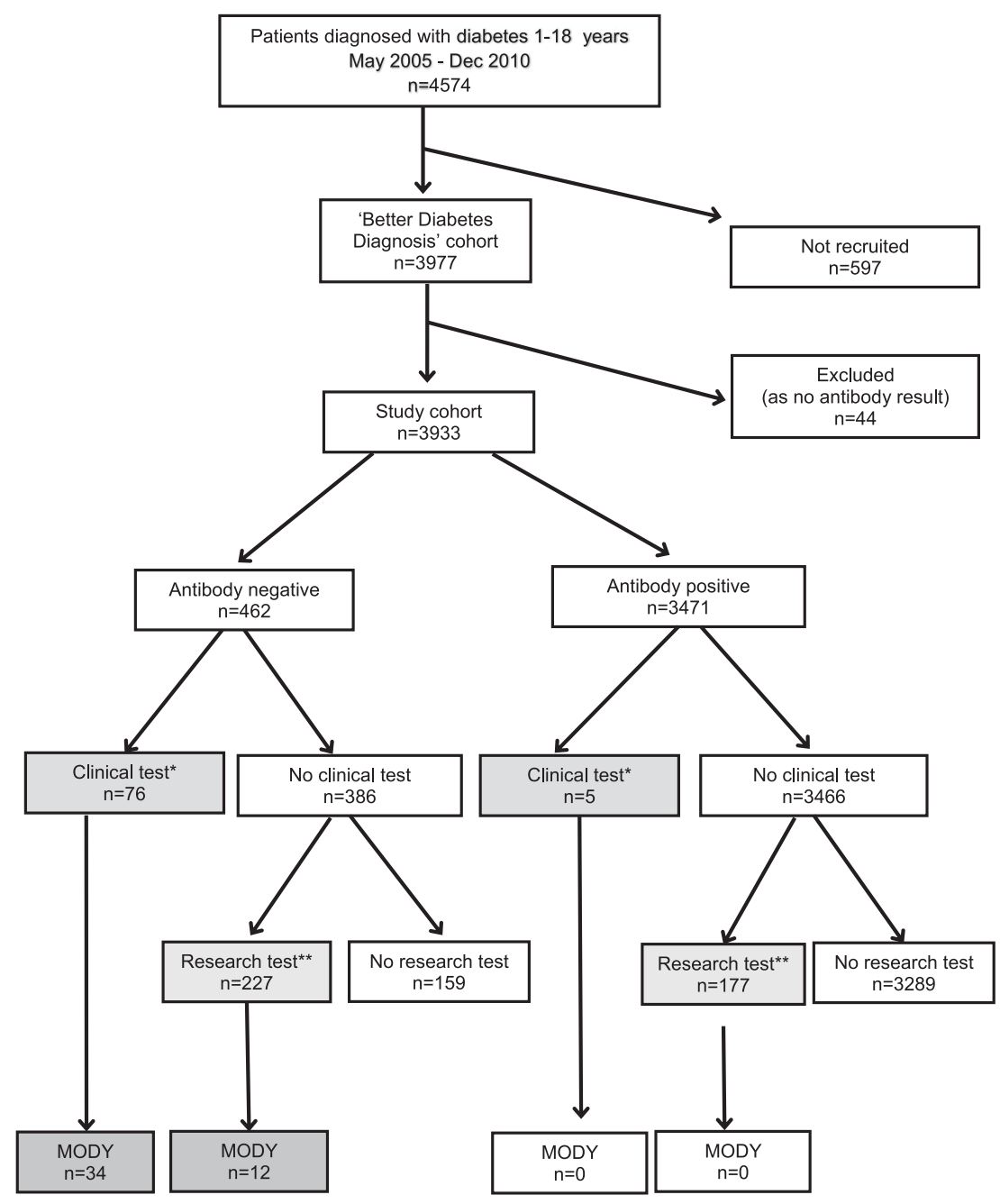

Figure 1-Study flow diagram. MODY was only identified in islet autoantibody-negative patients. All subjects were tested for GADA, IA-2A, ZnT8A, and IAA islet autoantibodies. Antibody positive was defined as at least one of these four autoantibodies being positive. The autoantibody results were fed back to the clinicians soon after diagnosis. The genetic tests are shown in the shaded boxes. *Genetic tests initiated by the clinician for MODY using routine diagnostic services are shown as "clinical test." **Genetic tests performed as part of the study to identify cases missed by clinical testing are defined as "research test."

\section{Details of Testing}

\section{Autoantibodies}

GADA, IA-2A, ZnT8A, and IAA autoantibodies were analyzed in radiobinding assays (23). The cutoff values used equated to the level found in only $1 \%$ of an age-matched population (23). GADA and IA-2A levels were expressed as units per milliliter derived from the World Health Organization standard 97/550 and were considered positive if GADA levels were $>35$ units $/ \mathrm{mL}$ and IA-2A levels $>5$ units $/ \mathrm{mL}$. The intra-assay coefficient of variation (CV) for duplicates was $5 \%$ for GADA and $11 \%$ for IA-2A. The radioligand binding assays for all three ZnT8A variants (ZnT8RA, ZnT8WA, and $Z n T 8 Q A$ ) were analyzed (24). Cutoff values for positive results were ZnT8RA $\geq 75$ units $/ \mathrm{mL}$, ZnT8WA $\geq 75$ units $/ \mathrm{mL}$, and
ZnT8QA $\geq 100$ units/mL. The intra-assay CV was 5.5\% for ZnT8RA, 5.3\% for ZnT8WA, and $4.9 \%$ for ZnT8QA. IAA was considered positive if it was $>0.8$ relative units. The intra-assay CV in the IAA assay was $6.0 \%$ (23).

The laboratory undertaking the autoantibody analyses participates in the biannual Islet Autoantibody Standardization Program (http://www.immunologyofdiabetessociety .com/).

\section{HLA Genotyping}

Dried blood spots were used for PCR amplification with sequence-specific oligonucleotide probes of HLA-DQB1 and DQA1 alleles using a DELFIA Hybridization assay (PerkinElmer, Boston, MA) (25). The HLA-DQB1* probes defined the presence of HLA-DQB1*02, 03:02, 03:01,
06:02, 06:03, and 06:04 alleles, and the HLA-DQA1 probes the DQA1*02:01, 03, and 05 alleles (25-27).

\section{C-peptide Measurement}

Serum C-peptide, from the random nonfasting blood samples, was measured at Linkoping University with a time-resolved fluoroimmunoassay (AutoDELFIA C-peptide kit; Wallac, Turku, Finland), with a detection level of $0.03 \mathrm{nmol} / \mathrm{L}$ (28).

\section{Molecular Genetic Testing for MODY}

The coding exons and conserved splice sites of HNF1A, HNF4A, and GCK were amplified by $P C R$ and sequenced on an $A B I$ 3730 (Applied Biosystems, Warrington, U.K.). Sequences were compared with the published reference sequences (NM_000545.6 for HNF1A, NM_175914.4 for HNF4A, and NM_000162.5 for GCK) using Mutation Surveyor v3.24 (SoftGenetics, State College, PA) or ABI SeqScape Software v2.5 (Applied Biosystems). Variants were classified according to the American College of Medical Genetics and Genomics guidelines (29). MODY was diagnosed by the identification of heterozygous pathogenic or likely pathogenic variants.

\section{Statistical Methods}

For statistical testing of binary variables, we used the Fisher exact test, and for continuous traits, we used unpaired $t$ tests. Where the continuous traits were not normally distributed, we log-transformed the variable and indicated this in the table legends. All analyses were performed in Stata v14.

\section{Ethics Approval}

The Regional Ethics Board at Karolinska Institute approved the BDD study (Dnrs 2004-826/1, 2006/1082-32, and 2009/ 1684-32)

The study is written in line with STrengthening the Reporting of OBservational studies in Epidemiology guidelines (https:// strobe-statement.org/) for cohort studies.

\section{RESULTS}

\section{Eighty-Eight Percent of Patients Were Positive for at Least One Islet Autoantibody}

When all four autoantibodies were analyzed, $88 \%(3,471$ out of 3,933$)$ of patients were positive for at least one islet autoantibody. The characteristics of the autoantibody-positive and autoantibodynegative patients are provided in Supplementary Table 1 . We examined how 
testing a lower number of autoantibodies would change the number of individuals identified as autoantibody negative (Table 1). Table 1 shows that each additional autoantibody tested resulted in fewer autoantibody-negative patients being identified, but with a reducing impact. Therefore, the number of autoantibodynegative patients is $49 \%$ with GADA testing only, $17 \%$ with GADA and IA-2A, $13 \%$ with GADA, IA-2A, and $\mathrm{ZnT} 8 \mathrm{~A}$, and $12 \%$ with all four autoantibodies. The detailed breakdown of the distribution of different autoantibodies is in Supplementary Fig. 1.

\section{MODY Was Only Identified in} Autoantibody-Negative Patients

There were no subjects with MODY identified in the autoantibody-positive patients tested either clinically $(n=5)$ or through research testing of consecutively selected individuals ( $n=177$ ) (Fig. 1).

\section{Clinical Features of Those With Confirmed MODY}

The strongest discriminatory clinical feature of MODY at diagnosis was being negative to all four islet autoantibodies (100\% vs. $11 \%$ not-known MODY; $P=2 \times$ $\left.10^{-44}\right)$. Other key discriminatory features were lower $\mathrm{HbA}_{1 \mathrm{c}}(7.0 \%$ vs. $10.7 \%$ [53 vs. $93 \mathrm{mmol} / \mathrm{mol}] ; P=1 \times 10^{-20}$ ), lower random plasma glucose (mean 11.7 vs. $\left.26.7 \mathrm{mmol} / \mathrm{L} ; P=3 \times 10^{-19}\right)$, parental diabetes ( $63 \%$ vs. $12 \%$; $P=1 \times 10^{-15}$ ), and not having DKA (0 out of 46 vs. 601 out of 3,887; $P=0.001$ ) (Table 2).

There Was a High Detection Rate of MODY, 34 out of 76 (45\%), in the Autoantibody-Negative Patients Tested on Clinician's Request Clinical molecular genetic diagnosis of MODY in these 34 patients was made at a median of 9 (interquartile range 4-21) months post-clinical diagnosis of diabetes
(Fig. 1). The patients clinically tested were more likely to be autoantibody negative, had lower plasma glucose and $\mathrm{HbA}_{1 \mathrm{c}}$, and were more likely to have a parent with diabetes (Supplementary Table 4).

There was a lower rate of MODY detection, 12 out of 227 (5\%), in the autoantibody-negative patients who had genetic testing as part of this research and were not referred for genetic testing by their clinicians (Fig. 1).

The MODY subtypes identified were: GCK MODY in $29(63 \%)$, HNF1A MODY in $10(22 \%)$, and HNF4A MODY in 7 (15\%) patients. The distribution of the different MODY types in the autoantibody-negative group, not referred for genetic testing by clinicians and tested on a research basis, was similar. The specific gene variants identified are shown in Supplementary Table 5.

\section{Of Autoantibody-Negative Patients, $15 \%$ Have MODY, Resulting in a Minimal Prevalence of $1.2 \%$}

When 303 autoantibody-negative patients were genetically tested, 46 had MODY (15\%). This equates to a minimal prevalence of $1.2 \%$ (46 out of 3,933 ) (Fig. 1). If we assume the detection rate of MODY in the 159 autoantibody-negative patients who were not tested on a research basis was $5 \%$ as seen in the 227 patients who were research tested, we would expect to find an additional 8 patients with MODY (Fig. 1). This gives an estimated prevalence of $1.4 \%$ (54 out of 3,933 ) in this pediatric population.

\section{Clinical Feature of MODY in Autoantibody-Negative Patients} Among the 462 autoantibody-negative patients, the 46 patients with MODY had markedly less severe hyperglycemia than those not known to have MODY. $\mathrm{HbA}_{1 \mathrm{c}}(7.0 \%$ vs. $10.2 \%$ [53 vs. $88 \mathrm{mmol}$ ) mol]) and mean random plasma glucose (11.7 vs. $23.7 \mathrm{mmol} / \mathrm{L}$ ) were less severe in the patients with MODY (Fig. 2 and Supplementary Fig. 2). They were less likely to have osmotic symptoms (polyuria and polydipsia) and weight loss but more likely to have a parent with diabetes ( $63 \%$ vs. $27 \%$; $P=4 \times 10^{-6}$ ) (Supplementary Table 6). In a multiple logistic regression model in this autoantibodynegative group of all variables that were significant in univariate analysis $(P<0.05)$, only plasma glucose $\left(P=6 \times 10^{-5}\right)$ and parental history of diabetes $(P=0.02)$ remained statistically significant discriminators of MODY. The characteristics of patients with GCK, HNF1A, and HNF4A MODY compared with patients with not-known MODY and with each other are shown in Supplementary Tables 7-9.

\section{Testing Using Previously Suggested Clinical Criteria for Discriminating MODY}

We went on to test how two previously defined criteria, relating to $\mathrm{HbA}_{1 \mathrm{c}}$ and family history, altered the detection rate and the number of patients identified in the 303 autoantibody-negative patients genetically tested for MODY (Supplementary Table 10). We used $\mathrm{HbA}_{1 c}$ rather than plasma glucose even though the latter was slightly more discriminatory, as it is used in previous clinical criteria and less variable than glucose.

If testing was limited to the 73 patients who, in addition to being autoantibody negative, also had an $\mathrm{HbA}_{1 \mathrm{c}}$ below the previously defined upper limit of $\mathrm{HbA}_{1 \mathrm{c}}$ $7.5 \%$ (58 $\mathrm{mmol} / \mathrm{mol}$ ) for the diagnosis of GCK MODY (30), this improved the detection rate to $49 \%$ (36 out of 73 ) and identified $78 \%$ (36 out of 46 ) of patients with MODY. These criteria, as expected, were excellent for detecting GCK MODY (29 out of 29 patients) but also detected

Table 1-The number of patients positive for autoantibodies varies with the number of autoantibodies tested at diagnosis in $B D D$, a national cohort of pediatric diabetes

\begin{tabular}{lccc} 
Number of autoantibodies tested & Autoantibody positive to: & $\begin{array}{c}N \text { positive (\%) } \\
\text { (out of 3,933) }\end{array}$ & $\begin{array}{c}N \text { (\%) of people who tested negative with this } \\
\text { testing of antibody combination }\end{array}$ \\
\hline One antibody & GADA & $2,081(53)$ & $1,852(47)$ \\
& IA-2A & $2,718(69)$ & $1,215(31)$ \\
Two antibodies & GADA and/or IA-2A & $3,263(83)$ & $670(17)$ \\
Three antibodies & GADA and/or IA-2A and/or ZnT8A & $3,428(87)$ & $505(13)$ \\
Four antibodies & GADA and/or IA-2A and/or ZnT8A & $3,471(88)$ & $462(12)$
\end{tabular}

Combinations of commonly tested autoantibodies, illustrating number and percentage of individuals positive to at least one autoantibody and percentage of patients antibody negative depending on which autoantibodies were tested. 


\begin{tabular}{|c|c|c|c|c|c|}
\hline \multirow[b]{2}{*}{ Phenotype } & \multicolumn{2}{|c|}{$\begin{array}{l}\text { HNF1A/HNF4A/GCK MODY } \\
\qquad(n=46)\end{array}$} & \multicolumn{2}{|c|}{$\begin{array}{l}\text { Not-known MODY } \\
(n=3,887) \wedge\end{array}$} & \multirow[b]{2}{*}{$P$} \\
\hline & $N$ & Mean $(S D)$ or $\%(N)$ & $N$ & Mean $(S D)$ or $\%(N)$ & \\
\hline \multicolumn{6}{|l|}{ Clinical features } \\
\hline Sex (female) & 46 & $54 \%(25)$ & 3,887 & $45 \%(1,730)$ & 0.23 \\
\hline Age at diagnosis (years), mean (SD) & 46 & $12.1(4.4)$ & 3,887 & $10.1(4.4)$ & $3 \times 10^{-3}$ \\
\hline Parental diabetes & 46 & $63 \%(29)$ & 3,887 & $12 \%(471)$ & $1 \times 10^{-15}$ \\
\hline Polyuria & 38 & $34 \%(13)$ & 3,570 & $95 \%(3,378)$ & $2 \times 10^{-22}$ \\
\hline Polydipsia & 38 & $34 \%(13)$ & 3,559 & $94 \%(3,350)$ & $2 \times 10^{-21}$ \\
\hline Weight loss & 38 & $16 \%(6)$ & 3,449 & $76 \%(2,619)$ & $1 \times 10^{-14}$ \\
\hline BMI (SD score), mean (SD) & 37 & $0.54(1.2)$ & 3,341 & $-0.36(1.6)$ & $7 \times 10^{-4}$ \\
\hline Acanthosis nigricans & 37 & $0 \%(0)$ & 3,453 & $1 \%(44)$ & 1 \\
\hline \multicolumn{6}{|l|}{ Investigations, early* } \\
\hline Plasma glucose (mmol/L), mean (SD) & 41 & $11.7(4.1)$ & 3,528 & $26.7(9.0)$ & $3 \times 10^{-19}$ \\
\hline $\mathrm{HbA}_{1 \mathrm{c}}(\%)$, mean (SD) & 46 & $7.0(3.7)$ & 3,495 & $10.7(4.5)$ & $1 \times 10^{-20}$ \\
\hline DKA & 46 & $0 \%(0)$ & 3,887 & $15 \%(601)$ & 0.001 \\
\hline \multicolumn{6}{|l|}{ Investigations, delayed ${ }^{\dagger}$} \\
\hline Four autoantibodies negative & 46 & $100 \%(46)$ & 3,887 & $11 \%(416)$ & $2 \times 10^{-44}$ \\
\hline High-risk HLA & 46 & $20 \%(9)$ & 3,830 & $70 \%(2,684)$ & $3 \times 10^{-12}$ \\
\hline C-peptide (nmol/L), mean (SD) & 41 & $0.99(0.63)$ & 3,555 & $0.34(0.43)$ & $1 \times 10^{-14}$ \\
\hline C-peptide $<0.2(\mathrm{nmol} / \mathrm{L})$ & 41 & $2 \%(1)$ & 3,555 & $40 \%(1,433)$ & $4 \times 10^{-8}$ \\
\hline
\end{tabular}

Data are $\%(N)$ unless otherwise indicated. Plasma glucose and C-peptide results based on $\log _{10}$ transformation. $\wedge$ Not-known MODY consists of $n=3,471$ autoantibody positive (182 MODY tested) and 416 autoantibody negative (257 MODY tested). *Early local testing with results $0-24 \mathrm{~h}$. tTesting at reference laboratories, where results were delayed (14-90 days).

$41 \%$ (7 out of 17 ) of patients with HNF1A and HNF4A MODY.

A dominant family history has been a defining feature of MODY (9). If testing was limited to patients who were autoantibody negative with a parental family history, this would result in testing

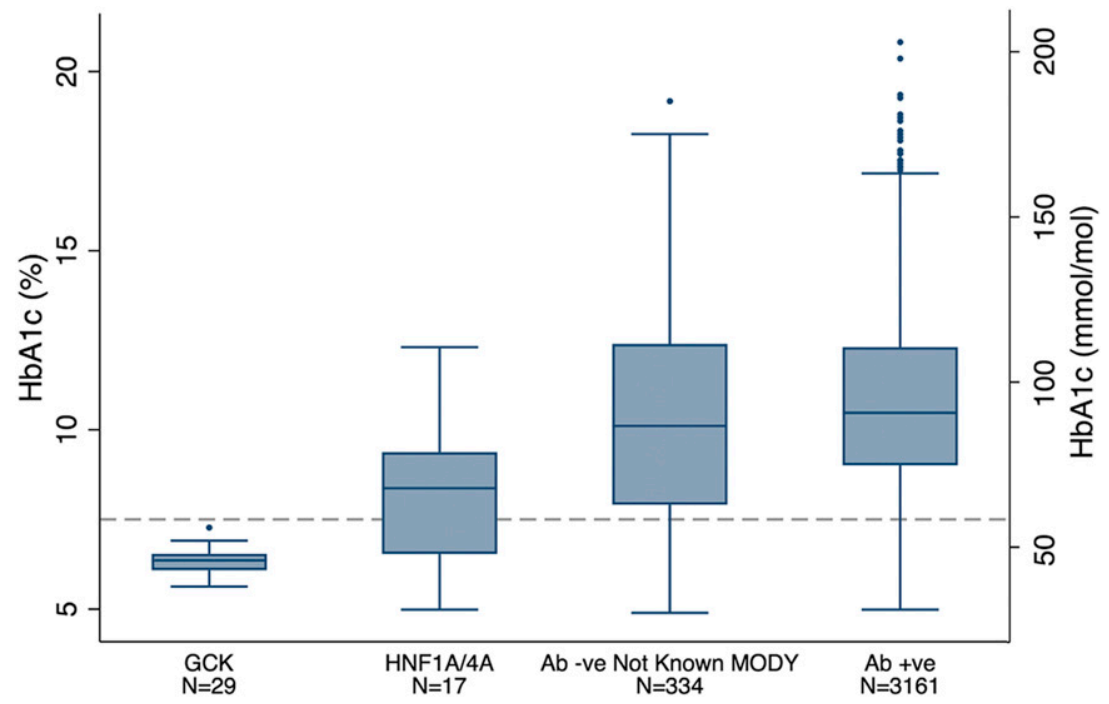

Figure 2-Patients with MODY had lower $\mathrm{HbA}_{1 \mathrm{c}}$ at diagnosis than those not known to have MODY. $\mathrm{HbA}_{1 \mathrm{c}}$ in patients with known MODY (both GCK and HNF1A/HNF4A) and those without known MODY (autoantibody negative [Ab -ve] and autoantibody positive [Ab + ve]). Data shown as a box and whisker plot: the top and bottom ends of the box are the upper and lower quartiles, respectively, and the median is marked as the horizontal line inside the box. The vertical lines indicate the maximum and minimum values excluding extreme outliers, shown as dots. $\mathrm{HbA}_{1 \mathrm{c}}$ of $7.5 \%$ (58 mmol/mol) cutoff for GCK MODY (30) shown as a dashed line. tested $(n=131)$, then the detection rate was $33 \%$ (44 out of 131), with 44 out of $46(96 \%)$ of case subjects with MODY detected.

Therefore, both glycemia at diagnosis and family history have a role in selecting which autoantibody-negative patients to test, but if selecting on a single clinical criteria, only then would $\mathrm{HbA}_{1 \mathrm{c}}<7.5 \%$ (58 $\mathrm{mmol} / \mathrm{mol}$ ) be both more sensitive and more specific than family history.

\section{Patients Identified With a Genetic Diagnosis of MODY Had an Excellent Outcome}

At a mean of 5.9 years after initial diabetes diagnosis, excellent glycemic control was achieved in all individuals with a genetic diagnosis of MODY with mean (SD) $\mathrm{HbA}_{1 \mathrm{c}} 6.4 \%$ (1.0\%) (47 [8] $\mathrm{mmol} / \mathrm{mol})$. A total of 42 out of 46 (91\%) patients were not on insulin and were on recommended treatment: no treatment for GCK MODY (29 out of $29 ; 100 \%)$ and diet or sulphonylurea for HNF1A MODY (9 out of 10; $90 \%$ ) and HNF4A MODY (4 out of 7; $57 \%$ ). A total of 14 out of 18 patients, started on insulin at initial diabetes diagnosis, had discontinued insulin treatment following a positive genetic test (Supplementary Table 11). 


\section{CONCLUSIONS}

This is the first large prospective national study to examine all clinical features at diagnosis of diabetes. Our study provides clear support for identifying pediatric patients for MODY testing by excluding type 1 diabetes through highquality, comprehensive autoantibody testing using four autoantibodies. In the autoantibody-negative patients, the most discriminatory clinical features are low glycemia (plasma glucose or $\mathrm{HbA}_{1 \mathrm{c}}$ ) and family history. Our study suggests that testing autoantibody-negative patients with $\mathrm{HbA}_{1 \mathrm{c}}<7.5 \%$ ( $<58 \mathrm{mmol} / \mathrm{mol}$ ) will identify $>75 \%$ of GCK, HNF1A, and HNF4A MODY with a detection rate of $\sim 50 \%$.

The minimal incidence of MODY in patients aged 1-18 years identified in this Swedish cohort was $1.2 \%$, and the estimated prevalence, if all autoantibodynegative patients had been tested in this cohort, would be $\sim 1.4 \%$. The prevalence seen in other studies of pediatric diabetes has been reported as follows: Norway $(0.5 \%)(3)$, U.S. (1.2\%) (1), Italy (1.6\% or $6.3 \%$ including incidental hyperglycemia) (5), Australia (1.9\%) (6), U.K. (2.5\%) (4), and Poland (3.1-4.2\%) (7). However, our study included a better coverage of the population ( $87 \%$ of all newly diagnosed case subjects with pediatric diabetes), and our data were collected prospectively, as opposed to a cross-sectional or selected cohort. Like the majority of pediatric studies, the most common etiology is GCK mutations. If patients with incidental hyperglycemia or impaired fasting glucose, but not diagnosed with diabetes, were included, the number of patients with GCK mutations would have been increased further (5).

Autoantibody negativity was a key feature of those identified with MODY. This finding was similar to previous studies, but within our study, autoantibody testing was comprehensive, with four autoantibodies tested, and also performed at diagnosis. This approach efficiently leads to more patients with type 1 diabetes being positively identified and reduces the number of case subjects needing consideration for MODY testing. The fact that our cohort was recruited at diagnosis means that a low ( $<200 \mathrm{pmol} / \mathrm{L})$ C-peptide result was not found in the majority of patients with autoantibodypositive type 1 diabetes. C-peptide testing was used in both U.S. and U.K. studies
$(1,4)$, but both recruited patients who were usually many years post-initial diabetes diagnosis.

Our study indicates that very few case subjects with MODY will be missed if genetic testing is limited to children who are negative for all four autoantibodies. As $1 \%$ of the normal population is isletautoantibody positive at the levels used as cutoffs, then $1 \%$ of patients with MODY can also be expected to have autoantibodies. However, as MODY is rare and type 1 diabetes is common in pediatric populations with diabetes, and $\sim 90 \%$ of children with type 1 diabetes are autoantibody positive close to diagnosis, the number of patients with MODY predicted in the autoantibodypositive patients would be $<0.1 \%$. Therefore, we consider data from this and other studies means autoantibody positivity is a reasonable exclusion for progressing to genetic testing in a person with diabetes in the pediatric age range $(14,31)$.

The breakdown of autoantibody positivity indicates that there is a clear benefit for testing three islet autoantibodies (GADA, IA-2A, and ZnT8A). However, only a few additional patients will be found to be positive if IAA testing is also performed at diagnosis. As testing IAA is technically difficult and only reduces the number of patients who are autoantibody negative from $13 \%$ of pediatric diabetes to $12 \%$, it may not be considered necessary clinically.

In our study, the results of the autoantibody tests were returned to all clinicians, and this was a major determinant of which patients the clinicians referred for genetic testing. This led to a very high rate of detection of MODY (45\%) compared with $27 \%$ in reported routine services (8).

This is the first study performed at the time of diabetes diagnosis. Samples and data were typically collected at diagnosis before insulin was given. The U.S. SEARCH for Diabetes in Youth (SEARCH) study included patients close to diagnosis but typically $\sim 8$ months post-initial diabetes presentation (1). Our study enabled accurate recording of initial symptoms and acute investigations at diagnosis of diabetes. The key features of those with MODY whom we identified at diabetes diagnosis included lower $\mathrm{HbA}_{1 \mathrm{c}}$, lower plasma glucose, and less osmotic symptoms, which all reflect less severe hyperglycemia, and there were no patients with MODY who presented in ketoacidosis.

A key issue is what is the appropriate level of sensitivity and specificity of the threshold set for systematic testing of patients. The low prevalence of MODY means that even features with a very high odds ratio have a low positive predictive value. This makes it hard to identify likely case subjects, and unduly strict criteria, while resulting in a high detection rate, will miss case subjects. With a reduction in the cost of genetic testing, it may be the most effective strategy to sequence all pediatric patients with diabetes who are negative on testing four autoantibodies, leading to a detection rate of $\sim 15 \%$. Our results support that prespecified cutoffs of $\mathrm{HbA}_{1 \mathrm{c}}<7.5 \%$ in autoantibody-negative patients give a much higher detection rate for the person being tested having MODY ( 50\%) but would miss $\sim 22 \%$ of case subjects with MODY (mainly HNF1A and HNF4A MODY). A compromise might be to test all autoantibody-negative patients with an $\mathrm{HbA}_{1 \mathrm{c}}<7.5 \%$ or a parent with diabetes; this approach had a detection rate $\sim 33 \%$ and detected $\sim 94 \%$ of patients with MODY.

It is interesting that, after receiving the islet autoantibody results, clinicians chose to test only 76 out of 462 (16\%) of the autoantibody-negative patients but had a high detection rate of 34 out of 76 (45\%) in those they did test. The main factors that influenced clinician testing were severity of glycemia and family history (Supplementary Table 12). Ultimately, a model that integrates all clinical factors may outperform clinician choice, but at present, either testing all islet autoantibody-negative patients or those with a predefined $\mathrm{HbA}_{1 \mathrm{c}}$ cutoff of $<7.5 \%$ at diagnosis performs better than clinician choice, with fewer case subjects with MODY missed.

It is important to detect MODY, as our results show improved outcome both in terms of insulin cessation and $\mathrm{HbA}_{1 \mathrm{c}}$. This study has prospectively followed up the long-term impact of a diagnosis of MODY from diabetes diagnosis to effect on clinical outcomes; 42 out of 46 (91\%) patients were not on insulin at follow-up, and 14 out of 18 of these had ceased insulin treatment (which had been started at initial diabetes diagnosis), and excellent glycemic control was 
achieved $\left(\mathrm{HbA}_{1 \mathrm{c}}\right.$ mean [SD] 6.4\% [1.0\%] [47 (8) $\mathrm{mmol} / \mathrm{mol}]$ ) (Supplementary Table 11). Two out of four of the individuals still on insulin chose to remain on this treatment. Stopping insulin can be a major challenge for some patients, and this highlights the importance of identifying the correct genetic diagnosis as soon as possible (32).

The major strengths of this study are that it is a large, consecutive series recruiting $87 \%$ of case subjects with newly diagnosed diabetes in the pediatric population, allowing assessment of both clinical features and autoantibodies at diagnosis.

It is also a strength that all autoantibody tests were carried out for the entire country at a central BDD laboratory at the Lund University Clinical Research Centre at Skåne University Hospital. The laboratory is participating in the Islet Autoantibody Standardization program and has very good results. In addition, all samples are subjected to end point titration to better define cutoff levels compared with a large number of serum and plasma samples from healthy individuals. All three isoforms of ZnT8A (R, W, or $Q$ at position 325$)$ were analyzed to ensure that children single positive for any of the three variants were accounted for $(15,23)$. However, most commonly clinically used ELISA assays for ZnT8A will detect as positive $>99 \%$ of samples positive for the three separate isoforms, as they detect the common $\mathrm{R}$ and $\mathrm{W}$ variants. Only pathogenic or likely pathogenic variants have been included in this study (Supplementary Table 5). The only likely pathogenic variants are in GCK, and therefore, further investigations could be performed by testing other family members to check for segregation of the variant with fasting hyperglycemia and raised $\mathrm{HbA}_{1 \mathrm{c}}$. Variants of uncertain significance were not included because they cannot be used to diagnose MODY.

A weakness of this study is that only the three most common subtypes of MODY that can alter treatment were tested. If a next-generation sequencing approach is used in children allowing testing of all case subjects with potential monogenic subtypes, then there is a slight increase $(\sim 15 \%)$ in case subjects with more monogenic diabetes than the common GCK, HNF1A, and HNF4A MODY alone (4). The data presented apply to the Swedish population and will vary in other populations, especially when there is a higher representation of ethnic groups with a lower prevalence of type 1 diabetes and higher prevalence of type 2 diabetes.

In conclusion, at diagnosis of pediatric diabetes, comprehensive autoantibody testing and degree of glycemia are key clinical features of MODY, allowing differentiation from type 1 diabetes. Establishing negativity to four islet autoantibodies at diabetes diagnosis in the pediatric population efficiently identifies which individuals should be considered for genetic testing. Within the autoantibodynegative patients, modest hyperglycemia, indicated by an $\mathrm{HbA}_{1 \mathrm{c}}<58 \mathrm{mmol} / \mathrm{mol}$ $(<7.5 \%)$, and family history are further features that can be used to guide testing. Identifying patients for genetic testing at diabetes diagnosis will prevent delays in the correct molecular genetic diagnosis of patients. This will lead to improvements in treatment and quality of life and reductions in treatment and monitoring costs and should be universally advocated in patients with pediatric diabetes at diagnosis.

Acknowledgments. The authors thank Gerald Hysenaj and Sam Hall, who carried out the molecular genetic testing for the research patients at Molecular Genetics, Royal Devon and Exeter NHS Foundation Trust, Exeter, U.K.; Anita Ramelius, Ida Jönsson, Rasmus Bennet, Ingrid Wigheden, and Zehlia Mestan, who carried out HLA typing and islet autoantibody measurements for all patients at the Department of Clinical Sciences, Lund University, Skåne University Hospital, Malmö, Sweden; and Ingela Johansson and Gosia Smolinska, who carried out the C-peptide determinations at the Department of Clinical and Experimental Medicine, Linköping University, Linköping, Sweden.

Funding. This study was supported by the Swedish Child Diabetes Foundation, the National Institutes of Health (DK-063861), National Institute for Health Research (U.K.) via funding for the Clinical Research Facility, and the Wellcome Trust. S.E. and A.T.H. are Wellcome Trust Senior Investigators (098395/Z/12/Z), and A.T.H. is a National Institute for Health Research Senior Investigator.

Duality of Interest. No potential conflicts of interest relevant to this article were reported. Author Contributions. A.C., M.S., S.E., A.L., G.F., L.G., J.L., C.M., and A.T.H. were all involved in the conception and design of this study. A.C., A.L., G.F., K.C., Q.B., C.V.-A., S.A.I., H.E.L., U.S., E.Ö., L.G., J.L., and C.M. provided data. A.C., S.E., M.W., Å.L., K.C., and E.Ö. analyzed data. A.C., M.S., S.E., M.W., A.L., K.C., J.L., C.M., and A.T.H. interpreted data. A.C., M.S., C.M., and A.T.H. wrote the drafts of the paper. All authors critically reviewed and modified the manuscript and have agreed to the final submitted version and to be accountable for the work. A.T.H. is the guarantor of this work and, as such, had full access to all of the data in the study and takes responsibility for the integrity of the data and the accuracy of the data analysis.

\section{References}

1. Pihoker C, Gilliam LK, Ellard S, et al.; SEARCH for Diabetes in Youth Study Group. Prevalence, characteristics and clinical diagnosis of maturity onset diabetes of the young due to mutations in HNF1A, HNF4A, and glucokinase: results from the SEARCH for Diabetes in Youth. J Clin Endocrinol Metab 2013;98:4055-4062

2. Hattersley AT, Greeley SAW, Polak M, et al. ISPAD Clinical Practice Consensus Guidelines 2018: the diagnosis and management of monogenic diabetes in children and adolescents. Pediatr Diabetes 2018;19(Suppl. 27):47-63

3. Johansson BB, Irgens HU, Molnes J, et al. Targeted next-generation sequencing reveals MODY in up to $6.5 \%$ of antibody-negative diabetes cases listed in the Norwegian Childhood Diabetes Registry. Diabetologia 2017;60:625-635

4. Shepherd M, Shields B, Hammersley S, et al.; UNITED Team. Systematic population screening, using biomarkers and genetic testing, identifies $2.5 \%$ of the U.K. pediatric diabetes population with monogenic diabetes. Diabetes Care 2016; 39:1879-1888

5. Delvecchio M, Mozzillo E, Salzano G, et al.; Diabetes Study Group of the Italian Society of Pediatric Endocrinology and Diabetes (ISPED). Monogenic diabetes accounts for $6.3 \%$ of cases referred to 15 Italian pediatric diabetes centres during 2007 to 2012. J Clin Endocrinol Metab 2017;102:1826-1834

6. Johnson SR, Ellis JJ, Leo PJ, et al. Comprehensive genetic screening: the prevalence of maturity-onset diabetes of the young gene variants in a population-based childhood diabetes cohort. Pediatr Diabetes 2019;20:57-64

7. Fendler $W$, Borowiec $M$, BaranowskaJazwiecka $A$, et al. Prevalence of monogenic diabetes amongst Polish children after a nationwide genetic screening campaign. Diabetologia 2012;55:2631-2635

8. Shields BM, Hicks S, Shepherd MH, Colclough K, Hattersley AT, Ellard S. Maturity-onset diabetes of the young (MODY): how many cases are we missing? Diabetologia 2010;53:2504-2508 9. Murphy R, Ellard S, Hattersley AT. Clinical implications of a molecular genetic classification of monogenic beta-cell diabetes. Nat Clin Pract Endocrinol Metab 2008;4:200-213

10. Pearson ER, Pruhova S, Tack CJ, et al. Molecular genetics and phenotypic characteristics of MODY caused by hepatocyte nuclear factor 4alpha mutations in a large European collection. Diabetologia 2005;48:878-885

11. Bellanné-Chantelot C, Lévy DJ, Carette C, et al.; French Monogenic Diabetes Study Group. Clinical characteristics and diagnostic criteria of maturity-onset diabetes of the young (MODY) due to molecular anomalies of the HNF1A gene. J Clin Endocrinol Metab 2011;96:E1346-E1351

12. NCD Risk Factor Collaboration (NCD-RisC). Worldwide trends in body-mass index, underweight, overweight, and obesity from 1975 to 2016: a pooled analysis of 2416 population-based measurement studies in $\mathbf{1 2 8 . 9}$ million children, 
adolescents, and adults. Lancet 2017;390:26272642

13. Kleinberger JW, Copeland KC, Gandica RG, et al. Monogenic diabetes in overweight and obese youth diagnosed with type 2 diabetes: the TODAY clinical trial. Genet Med 2018;20: 583-590

14. McDonald TJ, Colclough K, Brown R, et al. Islet autoantibodies can discriminate maturityonset diabetes of the young (MODY) from type 1 diabetes. Diabet Med 2011;28:1028-1033

15. Andersson C, Vaziri-Sani F, Delli A, et al.; BDD Study Group. Triple specificity of ZnT8 autoantibodies in relation to HLA and other islet autoantibodies in childhood and adolescent type 1 diabetes. Pediatr Diabetes 2013;14:97-105 16. Bravis V, Kaur A, Walkey HC, et al.; ADDRESS2 Management Committee, Patient Advocate Group and Investigators Relationship between islet autoantibody status and the clinical characteristics of children and adults with incident type 1 diabetes in a UK cohort. BMJ Open 2018;8: e020904

17. Bacon S, Kyithar MP, Rizvi SR, et al. Successful maintenance on sulphonylurea therapy and low diabetes complication rates in a HNF1AMODY cohort. Diabet Med 2016;33:976-984

18. Shepherd MH, Shields BM, Hudson M, et al.; UNITED study. A UK nationwide prospective study of treatment change in MODY: genetic subtype and clinical characteristics predict optimal glycaemic control after discontinuing insulin and metformin. Diabetologia 2018;61: 2520-2527

19. Naylor RN, John PM, Winn AN, et al. Costeffectiveness of MODY genetic testing: translating genomic advances into practical health applications. Diabetes Care 2014;37:202-209
20. Shields BM, McDonald TJ, Ellard S, Campbell MJ, Hyde C, Hattersley AT. The development and validation of a clinical prediction model to determine the probability of MODY in patients with young-onset diabetes. Diabetologia 2012;55: 1265-1272

21. Persson $\mathrm{M}$, Becker $\mathrm{C}$, Elding Larsson $\mathrm{H}$, et al. The Better Diabetes Diagnosis (BDD) study - a review of a nationwide prospective cohort study in Sweden Diabetes Res Clin Pract 2018;140:236-244

22. Hanberger L, Samuelsson U, Lindblad B, Ludvigsson J; Swedish Childhood Diabetes Registry SWEDIABKIDS. A1C in children and adolescents with diabetes in relation to certain clinical parameters: the Swedish Childhood Diabetes Registry SWEDIABKIDS. Diabetes Care 2008 31:927-929

23. Delli AJ, Vaziri-Sani F, Lindblad B, et al.; Better Diabetes Diagnosis Study Group. Zinc transporter 8 autoantibodies and their association with SLC30A8 and HLA-DQ genes differ between immigrant and Swedish patients with newly diagnosed type 1 diabetes in the Better Diabetes Diagnosis study. Diabetes 2012;61:2556-2564

24. Vaziri-Sani F, Delli AJ, Elding-Larsson H, et al. A novel triple mix radiobinding assay for the three ZnT8 (ZnT8-RWQ) autoantibody variants in children with newly diagnosed diabetes. J Immunol Methods 2011;371:25-37

25. Kiviniemi M, Hermann R, Nurmi J, et al.; TEDDY Study Group. A high-throughput population screening system for the estimation of genetic risk for type 1 diabetes: an application for the TEDDY (the Environmental Determinants of Diabetes in the Young) study. Diabetes Techno Ther 2007;9:460-472

26. Larsson HE, Lynch K, Lernmark B, Hansson G, Lernmark A, Ivarsson SA. Relationship between increased relative birthweight and infections during pregnancy in children with a high-risk diabetes HLA genotype. Diabetologia 2007;50: 1161-1169

27. Hagopian WA, Erlich $H$, Lernmark A, et al.; TEDDY Study Group. The Environmental Determinants of Diabetes in the Young (TEDDY): genetic criteria and international diabetes risk screening of 421000 infants. Pediatr Diabetes 2011;12:733-743

28. Ludvigsson J, Carlsson A, Forsander G, et al C-peptide in the classification of diabetes in children and adolescents. Pediatr Diabetes 2012;13:45-50

29. Richards S, Aziz N, Bale S, et al.; ACMG Laboratory Quality Assurance Committee. Standards and guidelines for the interpretation of sequence variants: a joint consensus recommendation of the American College of Medical Genetics and Genomics and the Association for Molecular Pathology. Genet Med 2015;17: 405-424

30. Steele AM, Wensley KJ, Ellard S, et al. Use of $\mathrm{HbA} 1 \mathrm{c}$ in the identification of patients with hyperglycaemia caused by a glucokinase mutation: observational case control studies. PLoS One 2013;8:e65326

31. Patel KA, Weedon MN, Shields BM, Pearson ER, Hattersley AT, McDonald TJ; UNITED study team. Zinc transporter 8 autoantibodies (ZnT8A) and a type 1 diabetes genetic risk score can exclude individuals with type 1 diabetes from inappropriate genetic testing for monogenic diabetes. Diabetes Care 2019;42: e16-e17

32. Shepherd M. Stopping insulin injections following genetic testing in diabetes: impact on identity. Diabet Med 2010;27:838-843 